\title{
OPERATORS WITH LEFT INVERSES SIMILAR TO THEIR ADJOINTS
}

S. M. PATEL

Abstract. The primary object of this paper is to show that if $T$ is a left invertible operator with a left inverse $T_{1}$ and if there exists an operator $S$ such that $T^{*}=S^{-1} T_{1} S$ and $0 \notin \operatorname{cl}(W(S))$, then $T$ is similar to an isometry.

An operator $T$ (a bounded linear transformation) on a Hilbert space $H$ is called an isometry if $T^{*} T=I$, that is, if there exists a left inverse $T_{1}$ of $T$ such that $T^{*}=T_{1}$. A unitary operator is called cramped if and only if its spectrum is contained in an arc of the unit circle with central angle less than $\pi \cdot \sigma(T), \pi_{00}(T), \sigma_{1}(\hat{T}), \operatorname{cl}(W(T))$ and $r(T)$ will denote the spectrum, the set of isolated points of $\sigma(T)$ that are eigenvalues of finite multiplicity, the left essential spectrum, the closure of the numerical range, and the spectral radius of $T$, respectively.

Before stating results we remark that these results are similar to those obtained by Williams [4].

In [3] U. N. Singh and Kanta Mangla proved that if $T$ is an invertible operator and if there exist an operator $S$ such that $T^{*}=S^{-1} T^{-1} S$ and $0 \notin \mathrm{cl}(W(S))$, then $T$ is similar to a unitary operator. This leads to the following generalization.

THEOREM 1. If $T$ is a left invertible operator with a left inverse $T_{1}$ and if there exists an operator $S$ such that $T^{*}=S^{-1} T_{1} S$ and $0 \notin \operatorname{cl}(W(S))$ then $T$ is similar to an isometry.

Proof. Since $0 \notin \mathrm{cl}(W(S)), 0$ can be separated from $\operatorname{cl}(W(S))$ by a half plane. If necessary, we may replace $S$ by $S e^{i \theta}$ for suitable $\theta$, so that this half plane lies strictly on the right of the imaginary axis. Let $A=$ $\left(S+S^{*} / 2\right)$; then $A$ is positive and invertible. Let $A^{1 / 2}$ denote the positive square root of $A$. Then $A^{1 / 2}$ is invertible. Now

$$
\begin{aligned}
T_{1} A T_{1}^{*} & =\frac{1}{2}\left(T_{1} S T_{1}^{*}+T_{1} S^{*} T_{1}^{*}\right) \\
& =\frac{1}{2}\left(S T^{*} T_{1}^{*}+T_{1} T S^{*}\right)=\frac{1}{2}\left(S+S^{*}\right)=A .
\end{aligned}
$$

Received by the editors July 17, 1972 and, in revised form, October 11, 1972 and February 9, 1973.

AMS (MOS) subject classifications (1970). Primary 47A10, 47B10.

Key words and phrases. Left inverse, cramped operator, normaloid, polar decomposition, numerical range, left essential spectrum.

c American Mathematical Society 1973 
Thus

$$
T_{1} A T_{1}^{*}=A
$$

Let $B=A^{1 / 2} T_{1}^{*} A^{-1 / 2}$. Then

$$
B^{*} B=\left(A^{1 / 2} T_{1}^{*} A^{-1 / 2}\right)^{*}\left(A^{1 / 2} T_{1}^{*} A^{-1 / 2}\right)=A^{-1 / 2}\left(T_{1} A T_{1}^{*}\right) A^{-1 / 2} .
$$

Using (1) we get $B^{*} B=I$. Since

$$
B=A^{1 / 2}\left(S^{*-1} T S^{*}\right) A^{-1 / 2}=\left(S^{*} A^{-1 / 2}\right)^{-1} T\left(S^{*} A^{-1 / 2}\right),
$$

$T$ is similar to an isometry.

The preceding theorem may not be true if $S$ is merely invertible, and it does not satisfy the condition $0 \notin \operatorname{cl}(W(S))$. This can be seen by the following

EXAmple 1. Let $H$ be a two-dimensional Hilbert space and $T$ be an operator on $H$ with the matrix

$$
\left[\begin{array}{cc}
2^{1 / 2} & 1 \\
1 & 2^{1 / 2}
\end{array}\right]
$$

Now if $S$ is the invertible operator with the matrix $\left[\begin{array}{rr}1 & 0 \\ 0 & -1\end{array}\right]$ then $T^{*}=$ $S^{-1} T^{-1} S$. However, $T$ cannot be similar to an isometry as its spectrum does not lie in the unit disc.

The following is the converse to Theorem 1.

THEOREM 2. If $T$ is similar to an isometry, then $T$ has a left inverse $T_{1}$ satisfying $T^{*}=S^{-1} T_{1} S$ for some operator $S$ with $0 \notin \operatorname{cl}(W(S))$.

Proof. Our hypothesis implies the existence of an invertible operator $R$ such that $R^{-1} T R$ is an isometry. If we put $S=R R^{*}$, then $0 \notin \mathrm{cl}(W(S))$ and $T_{1}=S T^{*} S^{-1}$ is a left inverse of $T$.

Our next result shows that a suitable restriction on $T$ in Theorem 1 guarantees $T$ to be an isometry.

THEOREM 3. Let $T$ be a left invertible normaloid operator satisfying the hypothesis of Theorem 1. If $T_{1}$ is also normaloid then $T$ is an isometry.

Proof. By Theorem $1, T$ and $T_{1}$ are similar to an isometry and coisometry respectively. Consequently, $r(T)=1$ and $r\left(T_{1}\right)=1$. Since $T$ and $T_{1}$ are normaloid, $\|T\|=1$ and $\left\|T_{1}\right\|=1$. Then for any $x$ in $H,\|x\|=\left\|T_{1} T x\right\| \leqq$ $\|T x\| \leqq\|x\|$. Thus $\|T x\|=\|x\|$ for every $x$ in $H$, which shows that $T$ is an isometry.

If $T_{1}$ is not normaloid in Theorem 3 , then $T$ may not be an isometry. We sketch the following example to illustrate this point. 
EXAMPLE 2. Let $\left\{e_{i}\right\}_{i=1}^{\infty}$ be an orthonormal basis for $H$. Define $T$ as follows: $T e_{1}=\frac{1}{2} e_{2}$ and $T e_{n}=e_{n+1}$ for $n \neq 1$. Then $T$ is a left invertible operator with left inverse $T_{1}$ defined as follows: $T_{1} e_{1}=0, T_{1} e_{2}=2 e_{1}$ and $T_{1} e_{n}=e_{n-1}$ for all $n \neq 1$ and $n \neq 2$. Clearly $T$ is normaloid. Since $\left\|T_{1}\right\|=2$ and $r\left(T_{1}\right)=1, T_{1}$ is not normaloid. Also it can be seen that $T$ is not an isometry. However, if $S$ is a diagonal operator with diagonal $\left\{\frac{1}{4}, 1,1\right.$, $1, \cdots\}$, then $T^{*}=S^{-1} T_{1} S$ and $0 \notin \operatorname{cl}(W(S))$.

THEOREM 4. Let $T$ be a left invertible operator with the right-handed polar decomposition UP, where $U$ is an isometry and $P$ is positive and invertible. Let $T_{1}$ be the left inverse of $T$ with the left-handed polar decomposition $P^{-1} U^{*}$. If, for a cramped unitary operator $V, T^{*}=V^{*} T_{1} V$, then $T$ is an isometry.

Proof. By our hypothesis,

$$
T^{*}=P U^{*}=V^{*}\left(P^{-1} U^{*}\right) V=\left(V^{*} P^{-1} V\right)\left(V^{*} U^{*} V\right) .
$$

Then the uniqueness of the right-handed polar decomposition yields $P=V^{*} P^{-1} V$. Since $V$ is cramped, by Theorem $3, P$ turns out to be a unitary operator. This, together with the positiveness of $P$, gives $P=I$; thus $T=U$. This proves the theorem.

The following result proved in [3] with different technique is an easy consequence of the preceding theorem.

COROLlaRy. If $T$ is an invertible operator such that $T^{*}=V^{*} T^{-1} V$ and $V$ is a cramped unitary operator, then $T$ is unitary.

Our next theorem can be regarded as an extension of Theorem 1.

THEOREM 5. Let $T$ be a left invertible operator with a left inverse $T_{1}$. If there exists an operator $S$ such that $T^{*}=S^{-1} T_{1}^{p} S, 0 \notin \mathrm{cl}(W(S))$ and $p$ is a nonnegative integer, then $\sigma(T)$ lies in the unit disc.

Proof. To prove the theorem, it will suffice to show that the nonzero points of the boundary, $\partial \sigma(T)$, of $\sigma(T)$ lie on the unit circle. Let $\lambda$ be a nonzero point of $\partial \sigma(T)$. Since $\partial \sigma(T)$ is contained in the approximate point spectrum of $T$, we can choose a sequence $\left\{x_{n}\right\}$ of unit vectors such that $\left\|(T-\lambda) x_{n}\right\| \rightarrow 0$ and so $\left\|\left(T_{1}-\lambda^{-1}\right) x_{n}\right\| \rightarrow 0$. Then

$$
\begin{aligned}
\left|\left(\lambda^{-p}-\lambda^{*}\right)\left\langle S^{-1} x_{n}, x_{n}\right\rangle\right| & =\left|\left\langle\left(T^{*}-S^{-1} T_{1}^{p} S-\lambda^{*}+\lambda^{-p}\right) S^{-1} x_{n}, x_{n}\right\rangle\right| \\
& =\left|\left\langle\left(T^{*}-\lambda^{*}\right) S^{-1} x_{n}, x_{n}\right\rangle-\left\langle S^{-1}\left(T_{1}^{p}-\lambda^{-p}\right) x_{n}, x_{n}\right\rangle\right| \\
& =\left|\left\langle S^{-1} x_{n},(T-\lambda) x_{n}\right\rangle-\left\langle\left(T_{1}^{p}-\lambda^{-\nu}\right) x_{n}, S^{*-1} x_{n}\right\rangle\right| \\
& \leqq\left\|S^{-1}\right\|\left\|(T-\lambda) x_{n}\right\|+\left\|S^{-1}\right\|\left\|\left(T_{1}^{p}-\lambda^{-p}\right) x_{n}\right\| .
\end{aligned}
$$


Since $\left\|\left(T_{1}-\lambda^{-1}\right) x_{n}\right\| \rightarrow 0,\left\|\left(T_{1}^{p}-\lambda^{-p}\right) x_{n}\right\| \rightarrow 0$ and hence

$$
\left|\left(\lambda^{-p}-\lambda^{*}\right)\left\langle S^{-1} x_{n}, x_{n}\right\rangle\right| \rightarrow 0 .
$$

Now $0 \notin \operatorname{cl}(W(S))$ implies $0 \notin \operatorname{cl}\left(W\left(S^{-1}\right)\right)$. Therefore $\lambda^{-p}=\lambda^{*}$. As $\lambda$ is nonzero, we get $|\lambda|=1$.

Although Theorem 5 is an extension of Theorem 1, it does not generalize it, for if $p=1$, the conclusion of the theorem cannot say whether $T$ is similar to an isometry. However, we have

THEOREM 6. Let $T$ be a left invertible operator with a left inverse $T_{1}$. If there exists a selfadjoint operator $S$ such that $T^{*}=S^{-1} T_{1}^{p} S$ where $0 \notin \mathrm{cl}(W(S))$ and $p$ is a nonnegative integer, then $T$ is similar to an isometry.

Proof. For $p=1$, the result follows from Theorem 1. Therefore, let us assume that $p \neq 1$. By our hypothesis, $S$ is positive and invertible. Let $S^{1 / 2}$ be the positive square root of $S$. Clearly $S^{1 / 2}$ is invertible. If we write $A$ and $B$ respectively for $S^{-1 / 2} T S^{1 / 2}$ and $S^{-1 / 2} T_{1} S^{1 / 2}$, then $B$ will be a left inverse of $A$ and $A^{*}=B^{p}$. Therefore $A^{*} A=B^{p-1}$. This shows that $B^{p-1}$ is invertible. Since $p \neq 1$, we conclude from the invertibility of $B^{p-1}$ that $B$ is invertible. Thus $A^{*}=A^{-p}$. Now one can easily see that $A$ is normal and hence $\|A\|_{1}=\left\|A^{-p}\right\|=\left\|A^{-1}\right\|^{p}$. It follows from Theorem 5 that $\sigma(T)$ and hence $\sigma(A)$ lies in the unit disc. In consequence, $\|A\|=1$. Thus $1=\|A\|=\left\|A^{-1}\right\|$. These conditions imply that $A$ is unitary as in the proof of Theorem 3. This finishes the proof of our theorem.

In an attempt to generalize Theorem 3, we have

THEOREM 7. Let $T$ be a left invertible contraction with a left inverse $T_{1}$. If $T_{1}$ is normaloid and if $T^{*}=S^{-1} T_{1}^{\nu_{1}} T_{1}^{* p_{2}} S, 0 \notin \mathrm{cl}(W(S))$, and $p_{1}, p_{2}$ are nonnegative integers, then $T$ is an isometry.

Proof. Since $T_{1}$ is normaloid, there exists $\lambda \in \sigma\left(T_{1}\right)$ such that $|\lambda|=\left\|T_{1}\right\|$. Then there exists a sequence $\left\{x_{n}\right\}$ of unit vectors such that $\left\|\left(T_{1}-\lambda\right) x_{n}\right\| \rightarrow 0$. It is easy to show that $\left\|\left(T_{1}^{*}-\lambda^{*}\right) x_{n}\right\| \rightarrow 0$ and hence $\left\|\left(T^{*}-\lambda^{*-1}\right) x_{n}\right\| \rightarrow 0$. As argued before, it can be shown that $\lambda^{*-1}=$ $\lambda^{p_{1} \lambda * p_{2}}$. As $\lambda \neq 0$, we have $1=|\lambda|=\left\|T_{1}\right\|$. Now by our hypothesis $\|T\| \leqq$ 1 ; thus $T$ is an isometry.

Our final result is parallel to Theorem 1.1 proved in [1].

THEOREM 8. If $T$ is a left invertible operator with left inverse $T_{1}$ and if $T^{*}=S^{-1} T_{1}^{p} S+K, 0 \notin \mathrm{cl}(W(S)), p$ is a nonnegative integer and $K$ is a compact operator, then the following assertions hold:

1. If $\pi_{00}(T)=\varnothing$, then $\sigma(T)$ lies in the unit disc.

2. The set of all points of $\sigma(T)$ lying outside the unit disc is finite or countable. 
Proof. 1. Since $\partial \sigma(T) \subseteq \sigma_{1}(\hat{T}) \cup \pi_{00}(T)$ [2] and $\pi_{00}(T)=\varnothing$, then $\partial \sigma(T) \subseteq \sigma_{1}(\hat{T})$. Let $\lambda$ be a nonzero complex number in $\partial \sigma(T)$. Then by the definition of $\sigma_{1}(\hat{T})$, we find a sequence $\left\{x_{n}\right\}$ of unit vectors having the property that $x_{n} \rightarrow 0$ weakly and $\left\|(T-\lambda) x_{n}\right\| \rightarrow 0$. Since $K^{*}$ is compact, $x_{n} \rightarrow 0$ weakly implies that $\left\|K^{*} x_{n}\right\| \rightarrow 0$. By a slight modification in the proof of Theorem 5, it can be shown that $|\lambda|=1$. This completes the proof of the first assertion.

2. Since $\pi_{00}(T)$ is finite or countable and $\sigma_{1}(\hat{T})$ lies in the unit disc, as can be seen from the proof of the first assertion, it follows that a finite or countable number of points of $\partial \sigma(T)$ will be outside the unit disc and so the second assertion follows.

Remark. If $p=1$ in Theorem 8 , then $T$ will be similar to the sum of an isometry and a compact operator and hence the first assertion and the second one will follow. To see this, use the argument applied in the proof of Theorem 1 to show that $B^{*} B=I+K$ where $B$ is an operator defined in Theorem 1 and $K$ is a compact one. Since $B=U P$, where $U$ is an isometry and $P=\left(B^{*} B\right)^{1 / 2}=I+$ compact operator, we conclude that $B$ is the sum of an isometry and a compact operator. This shows that $T$ is similar to the sum of an isometry and a compact operator.

The author wishes to thank Dr. B. S. Yadav and also the referee for their suggestions of improvements over the original text of the paper.

\section{REFERENCES}

1. V. Istrățescu and I. Istrățescu, On some classes of operators. II, Math. Ann. 194 (1971), 126-134. MR 44 \#7351.

2. C. R. Putnam, The spectra of operators having resolvent of first order growth, Trans. Amer. Math. Soc. 133 (1968), 505-510. MR 37 \#4651.

3. U. N. Singh and Kanta Mangla, Operators with inverses similar to their adjoints, Proc. Amer. Math. Soc. 38 (1973), 258-260.

4. J. P. Williams, Operators similar to their adjoints, Proc. Amer. Math. Soc. 20 (1969), 121-123. MR 38 \#1552.

Faculty of Mathematics, University of Delhi, Delhi-7, India 